\section{Commentary: Coronavirus disease 2019 and acute aortic dissection: So many questions, so few answers}

\author{
Michael W. A. Chu, MD, MEd, ${ }^{\mathrm{a}}$ \\ Maral Ouzounian, MD, PhD, ${ }^{b}$ \\ Ismail El-Hamamsy, $\mathrm{MD}, \mathrm{PhD},{ }^{\mathrm{c}}$ and \\ Mark D. Peterson, MD, PhD, ${ }^{\mathrm{b}, \mathrm{d}}$ on behalf of the \\ Canadian Thoracic Aortic Collaborative
}

The coronavirus disease 2019 (COVID-19) pandemic has challenged our ability to deliver safe, timely, and highquality care for our patients with acute aortic dissection while protecting our highly specialized aortic team members. The pandemic has influenced the clinical, educational, and administrative activities of cardiac surgery units globally. Furthermore, our ability to effectively treat patients has been severely affected. This has translated into a dramatic reduction in elective aneurysm repairs and increased wait times with consequent potential harm to patients. The pandemic has also affected urgent surgical care: We suspect that patients have avoided seeking hospital care due to fear of acquiring COVID-19, resulting in reduced demand for emergency dissection repair. This is corroborated by an increase in the number of patients dying out of hospital, and an overall excess mortality seen in several jurisdictions during the pandemic not directly attributable to COVID-19 infections. ${ }^{1}$

Murana and colleagues ${ }^{2}$ from Bologna share their heroic experience with managing patients with acute aortic dissection early during the first wave of the pandemic through a national lockdown. They report patient avoidance behaviors

\footnotetext{
From the ${ }^{a}$ Division of Cardiac Surgery, Department of Surgery, Western University, London, Ontario, Canada; ${ }^{\mathrm{b}}$ Division of Cardiac Surgery, Department of Surgery, University of Toronto, Toronto, Ontario, Canada; ${ }^{\mathrm{c} D i v i s i o n}$ of Cardiac Surgery, Department of Surgery, Mount Sinai Hospital and Icahn School of Medicine at Mount Sinai, New York, New York; and ${ }^{\mathrm{d}}$ Division of Cardiac Surgery, Department of Surgery, St Michael's Hospital, Toronto, Ontario, Canada.

Disclosures: The authors reported no conflicts of interest.

The Journal policy requires editors and reviewers to disclose conflicts of interest and to decline handling or reviewing manuscripts for which they may have a conflict of interest. The editors and reviewers of this article have no conflicts of interest.

Received for publication Oct 21, 2020; revisions received Oct 21, 2020; accepted for publication Oct 23, 2020; available ahead of print Oct 29, 2020.

Address for reprints: Michael W. A. Chu, MD, MEd, Western University, B6-106 University Hospital, London Health Sciences Centre, 339 Windermere Rd, London, Ontario, N6A 5A5 Canada (E-mail: Michael.Chu@lhsc.on.ca).

JTCVS Techniques 2020;4:55-6

2666-2507

Copyright (C) 2020 The Authors. Published by Elsevier Inc. on behalf of The American Association for Thoracic Surgery. This is an open access article under the CC BY-NCND license (http://creativecommons.org/licenses/by-nc-nd/4.0/).

https://doi.org/10.1016/j.xjtc.2020.10.039
}

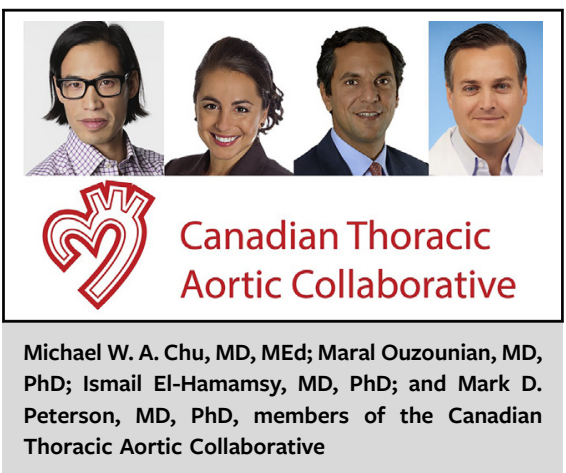

CENTRAL MESSAGE

COVID-19 has complicated pa-

tient care in acute aortic dissection, requiring a thoughtful team approach that is individualized for each patient.

causing delays in diagnosis, late presentations with malperfusion, and several patients who died before undergoing definitive repair. They were fortunate to have rapid testing protocols to confirm COVID-19 negative status on all patients undergoing operations who ultimately had very good outcomes with surgical repair. Although the authors observed pulmonary infiltrates on preoperative computed tomography scans that were concerning for COVID-19 in $30 \%$ of their patients, none of them tested positive for COVID-19.

Patients with acute aortic dissection will remain a challenging group to treat during this ongoing pandemic. Timely access to emergency surgical care remains a life-saving priority; however, several considerations need to be made.

- Rapid point-of-care COVID-19 testing should be systematically implemented to guide decision making for emergency operations and protect the surgical team and determine personal protective equipment requirements. COVID-19-positive patients undergoing cardiovascular surgery have an extremely high risk for perioperative death $^{3}$ and anecdotal reports of acute dissection repair suggest similarly poor outcomes.

- When taking a COVID-19-positive or suspect patient to the operating room for acute type A aortic dissection repair, operative expedience is likely paramount. Strategies to simplify surgical repair and minimize circulatory arrest and myocardial ischemic times should be considered. In this scenario, the primary focus must be on perioperative survival, even at the cost of late reoperation. 
- Lastly, if patients are COVID-19-positive, it would be wise to discuss the likelihood for postoperative respiratory failure and the potential need for aggressive respiratory support, including prolonged mechanical ventilation, tracheostomy, proning, and extracorporeal membrane oxygenation. Palliation may be considered in patients who are COVID-19 positive with extremely high-risk features (eg, octogenarians and patients with malperfusion syndrome) or hemodynamic instability.

Patients with acute aortic dissection remain challenging at the best of times. COVID-19 has complicated patient care significantly, requiring a very careful and thoughtful team approach that is individualized for each patient, considering local pandemic activity and resources. These patients deserve our very best efforts, while balancing the needs of the pandemic as a whole.

\section{References}

1. El-Hamamsy I, Brinster DR, DeRose JJ, Girardi LN, Hisamoto K, Imam MN, et al. The COVID-19 pandemic and acute aortic dissections in New York: a matter of public health. J Am Coll Cardiol. 2020;76:227-9.

2. Murana G, Folesani G, Botta L, Di Marco L, Leone A, Loforte A, et al. The effect of the coronavirus disease 2019 lockdown on type A acute aortic dissection: insights from Bologna. J Thorac Cardiovasc Surg Tech. 2020;4:50-4.

3. COVIDSurg Collaborative. Mortality and pulmonary complications in patients undergoing surgery with perioperative SARS-CoV-2 infection: an international cohort study. Lancet. 2020;396:27-38. 